\title{
Measurement of Release Wave Speed in Shock-Compressed Polycrystalline Alumina and Aluminum
}

\author{
Stephan J. Bless ${ }^{1}$ and Thomas J. Ahrens
}

Seismological Laboratory, California Institute of Technology, Pasadena, California 91125

\begin{abstract}
Values of release wave speed in shock-compressed polycrystalline alumina and aluminum (2024) have been measured to 400 and $260 \mathrm{kbar}$, respectively. These values, as well as previous data for aluminum and iron, have been compared with fourth-order finite strain extrapolations of the longitudinal sound speed $V_{p}$. For alumina in the pressure range 200-300 kbar and for $\alpha$ iron and $\epsilon$ iron the observed release wave speeds fall below any reasonable extrapolation of $V_{p}$. In the range 360-400 kbar the data for alumina indicate that $M\left(\partial^{2} M / \partial P^{2}\right) \simeq-50$ (where $M$ and $P$ are longitudinal modulus and pressure, respectively); this value is in approximate agreement with Graham's $\sim 200$-kbar data on elastic shock compression of single-crystal aluminum oxide. For aluminum the data indicate a value for $M\left(\partial^{2} M / \partial P^{2}\right)$ of $2.7 \pm 5.7$.
\end{abstract}

\section{INTRODUCTION}

Although the mean compressional and shear wave velocities as functions of depth are known to within relatively small uncertainties $(<1 \%)$ for certain regions of the earth's interior, such as the lower mantle, our ability to interpret these data in terms of mineralogy and temperature has been hampered by the lack of direct experimental data specifying the shear properties of silicates and oxides at very high pressure and of a validated theory for extrapolating these properties from lowpressure data. Direct measurements of longitudinal and shear velocities and their pressure and temperature derivatives have been carried out simultaneously at pressures of up to $10 \mathrm{kbar}$ 'and temperatures of $\sim 600^{\circ} \mathrm{C}$ [e.g., Spetzler, 1970] and at higher temperatures at ambient pressures [Soga and Anderson, 1967]. In order to extrapolate these results to high pressure or to reduce the properties of the earth to ambient pressure, formulations are usually applied on the basis of finite strain theory [e.g., Birch, 1952; Ahrens, 1972; Burdick and Anderson, 1975] or lattice theory [Sammis, 1972]. Comparison with shock wave data provides a critical series of tests to determine the range of compressions over which the low-pressure elastic moduli and their pressure and temperature derivatives may be reliably applied and to determine the relative merits of various formulations of finite strain theory. The pressure dependence of the bulk modulus has been studied by Anderson [1967] and later by Ahrens and Thomsen [1972], who compared the density observed in shock experiments with that predicted by several forms of finite strain theory for a wide class of materials. Because of the lack of similar data which may be used to specify the high-pressure moduli upon which the longitudinal and shear wave velocities depend, tests of the ability of finite strain theory to extrapolate seismic speeds validly have been sparse. Some high-pressure longitudinal elastic wave velocity data are reported by Erkman and Christensen [1967], Kusubov and van Thiel [1969a, b], Grady et al. [1975], and Anderson [1972] for 2024 aluminum, $\mathrm{SiO}_{2}$, and $\mathrm{NaCl}$.

The experiments reported in this paper were meant to provide new data on the longitudinal sound speed at high pressure in polycrystalline alumina $\left(\mathrm{Al}_{2} \mathrm{O}_{3}\right)$ in the 200 - to 400 -kbar range. This material is well suited for an evaluation of the finite strain theories, since although it is rather incompressible,

\footnotetext{
${ }^{1}$ Now at University of Dayton Research Institute, Dayton, Ohio 45469 .
}

Copyright (C) 1976 by the American Geophysical Union. its low-pressure elastic moduli and their pressure and temperature derivatives are well determined [Schreiber and Anderson. 1966], it possesses the geophysically important corundum structure, and it does not undergo phase changes in the pressure region under investigation. In addition, dense polycrystalline alumina is known to support a shear stress in the 30 - to 40-kbar range when it is shocked to states up to $\sim 300$ kbar [Ahrens et al., 1968] and hence is a candidate material for yielding data and high-pressure shear properties.

\section{EXPERIMENT}

In our experiments, very high pressures associated with uniaxial strain deformation are produced by the impact of a propellant-gun-launched flyer plate onto a target block. Both plate and block are of the same material, Lucalox (General Electric Corporation), a nonporous polycrystalline alumina (corundum), previously studied ultrasonically by Schreiber and Anderson [1966] and under shock conditions by Ahrens et al. [1968].

The target block (Figure 1) is wedge shaped, as in the experimental arrangement proposed by Fowles [1960]. Its thickness $L$ varies with position $y$ across the impact surface. The value of the wedge angle $\alpha$ is chosen such that the velocity of the contact point between the deformational shock and the target free surface greatly exceeds the rarefaction wave velocity in the target. A mirror consisting of a glass prism with a silvered surface is mounted behind the target. The prism is illuminated by a flash lamp and observed by a streak camera. The prism angle $\beta$ is determined such that internal reflection of light at the mirror surface occurs at nearly normal incidence.

An $(x, t)$ diagram which illustrates the shock and rarefaction waves which result from a given impact is shown in Figure 2. There the origin represents the collision between the flyer plate of thickness $a$, which is moving with velocity $u_{p}$, and the target, which is initially at rest. The pressure and the particle velocity produced by the impact are propagated as shock waves into the target and the flyer plate. Lines drawn from the origin represent the 'elastic shocks' (propagation speed $U_{1}$, particle velocity $u_{1}$ ) behind which the stress is assumed to be at the level of the Hugoniot elastic limit (HEL) (80-110 kbar for Lucalox) of the material. The deformational, or 'plastic,' shock waves follow the elastic shocks and propagate with speed $U_{2}$ with respect to undisturbed material; the particle velocity behind the deformational shocks, defined with respect to stationary coordinates, is $u_{2}$. At points 1 and 2 , release 


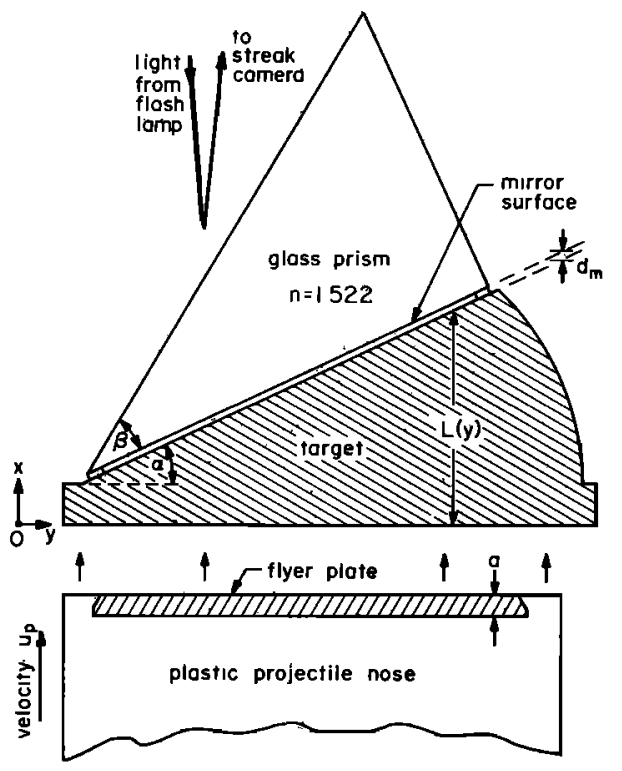

Fig. 1. Diagram of target geometry.

(rarefaction) waves are generated. As is the case upon loading, unloading is also usually assumed to take place elastically until the shear strength of the shock-compressed material is exceeded. If this elastic-plastic release theory applies and if $\partial V_{p} / \partial P>0$, where $V_{p}$ is the longitudinal elastic wave speed and $P$ is the pressure, then the velocity of the leading release wave characteristic, $c$, is equal to $V_{p}$ in the shock-compressed material [e.g., Zel'dovich and Raizer, 1966]. If the material displays no shear strength upon release, $c=v_{b}$, where $v_{b}$ is the bulk sound speed, equal to $(K / \rho)^{1 / 2}, K$ and $\rho$ being the adiabatic bulk modulus and density, respectively.

The release wave from point 1 overtakes the deformational shock at point 8 . The experiments which we have conducted were designed to measure the mean speed of the release wave over the path $1 \rightarrow 8$, by experimentally determining the distance $x_{8}$. Figure 2 is drawn for the case of target thickness $L>$ $x_{\mathrm{a}}$.

Figures $3 a$ and $3 b$ illustrate a typical experimental record. Figure $3 a$ shows the target prior to impact, superimposed upon an image of a slit through which the target is viewed with a streak camera during the experiment. Figure $3 b$ shows the streak photograph produced as the image of the slit is swept across the film plane during the experiment. The sweep rate is determined separately in a test using a Pockels-cell-modulated laser beam [Ahrens et al., 1971].

Because previous results are available for 2024 aluminum [Kusubov and van Thiel, 1969a, b; Erkman and Christensen, 1967], we first conducted two test experiments with that material.

\section{ANALYSIS OF THE EXPERIMENTS}

The apparent shock speed $U$, defined as the $x$ component of the velocity with which the reflecting surface of the prism is destroyed, is computed from

$$
U=\frac{W}{M} \frac{\sin \alpha \sin \beta}{\cos (\alpha+\beta)} \frac{1}{\tan \omega-\tan \gamma^{\prime}}
$$

where

$$
\tan \gamma^{\prime}=\cos (\alpha+\beta) \tan \gamma /(\cos \alpha \cos \beta)
$$

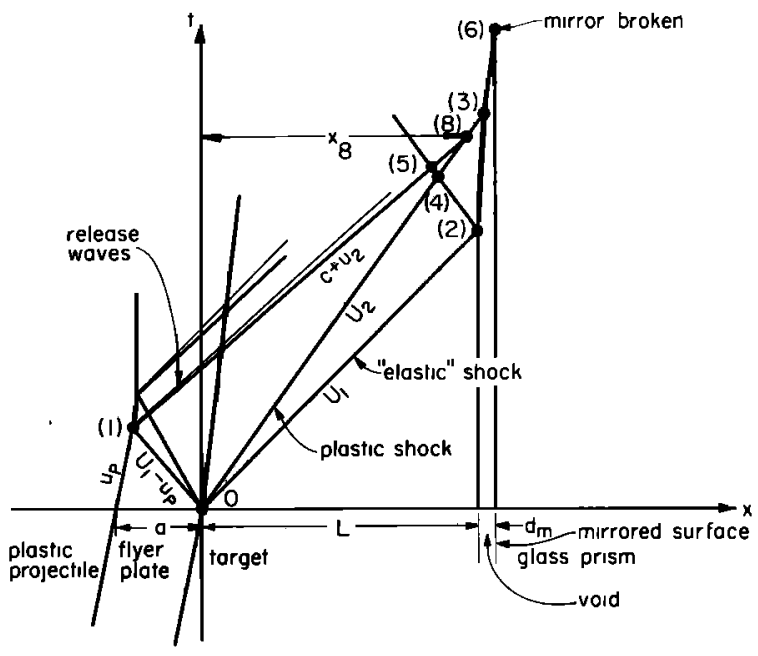

Fig. 2. An $(x, t)$ diagram for an experiment in which $L<L^{*}$.

and $\alpha$ and $\beta$ are the target angles, defined in Figure $1 . W$ and $M$ are the writing rate and magnification of the streak photograph; $\gamma$ is the tilt, measured on the streak photograph by means of the flat reference mirrors on the target (Figure 3a); and $\omega$ is the angle of the streak cutoff $(\omega=0$ and $\gamma=0$ represent simultaneity). The angle $\gamma^{\prime}$ is the angle of tilt of the shock front as it would be seen on the photograph if it were viewed through the prism.

Each point $\left(x_{m}, y_{m}\right)$ (referred to the $(x, y)$ axis in Figure 1) on the mirror surface lies above a thickness $L=x_{m}-d_{m}$ of the target and corresponds to a line on the streak record. Let $y^{\prime}$ be the coordinate measuring the transverse direction across the streak photograph. The distance $\Delta x_{m}$ between two points on the mirror surface which appear a distance $\Delta y^{\prime}$ apart on the streak photograph is given by

$$
\Delta x_{m}=\Delta y^{\prime} \sin \alpha \cos \beta /[M \cos (\alpha+\beta)]
$$

Reference lines are provided on the target shown in Figure $3 a$ in order to facilitate calculation of the target thickness which corresponds to a reference value of $y^{\prime}$.

The apparent shock speed $U$ is a function of $y^{\prime}$, since the rate at which the mirror is destroyed is a function of $L$. In particular, three regions can be distinguished on most streak records (such as Figure $3 b$ ).

1. Region 1 corresponds to $L<L^{* *}$; the rarefaction wave does not overtake either the free surface or the deformational shock before the mirror is broken. The apparent shock speed is independent of $L$. At $L=L^{* *}$ the rarefaction wave arrives at the free surface just as it contacts the mirror.

2. Region 2 corresponds to $L^{*}>L>L^{* *}$; the free surface is overtaken by the rarefaction wave between points 3 and 6 in Figure $2 . U$ has a relatively small value, since the time required by the free surface to cross the mirror gap increases with $L$. At $L=L^{*}$ the rarefaction wave overtakes the deformational shock just as it arrives at point 3 in Figure 2; i.e., points 3 and 8 coincide.

3. Region 3 corresponds to $L>L^{*}$; the rarefaction wave overtakes the deformational shock before the shock reaches the target free surface. $U$ will be a slowly varying function of $L$. This is the case for which Figure 2 is drawn.

Determination of $L^{*}$ and $L^{* *}$ from the streak records consititutes the basis for the calculation of the rarefaction wave speed $c$. Consider an $(x, t)$ diagram (drawn for a plane perpendicular to the impact surface) for an experiment in which 

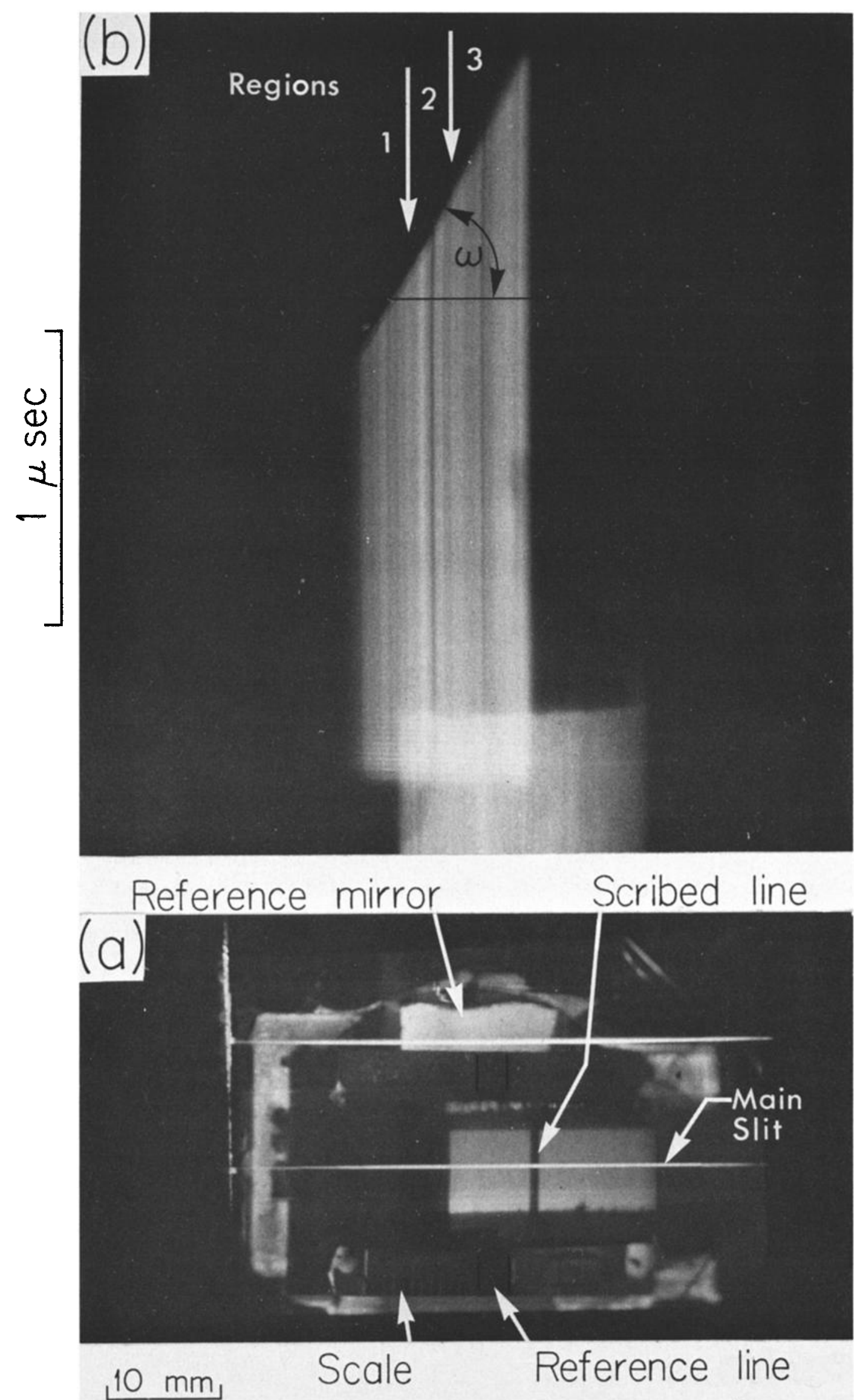

Fig. 3. (a) Image of target from shot A333, superimposed upon image of slit. Height of the reference lines scribed on the mirror can be determined from their relationship to the painted lines on the target, seen through the unsilvered portion of the glass prism. (b) Streak record from shot A333. Regions 1, 2, and 3 are discussed in the text. 
$L^{*}>L>L^{* *}$; the following points, $\left(x_{i}, t_{i}\right)$, can be defined: origin 0 , the locus of the impact; point 1 (e.g., $\left(x_{1}, t_{1}\right)$ ), the arrival of the elastic shock at the back surface of the flyer plate; points 2,3 , and $3^{\prime}$, the arrival of the elastic and deformational shocks from 0 and the release wave originating at point 1 at the free surface of the target, respectively; and points 4 and 5 , the intersections of the release wave reflected from point 2 with the deformational shock from 0 and the release wave from point 1 , respectively. The following additional points will occur on an $(x, t)$ diagram for an event in which $L$ is slightly smaller than $L^{* *}$ : point 6 , at which the free surface, moving from point 3 , arrives at the stationary mirror surface; and point 7 , at which the release wave through point 5 arrives at the free surface. For $L$ slightly larger than $L^{*}$, point 8 , at which the rarefaction wave from 1 overtakes the deformational shock, occurs (see Figure 2).

The following assumptions are made regarding the velocities of the characteristics or particle trajectories, $U_{i j}$, connecting each pair of points $i$ and $j$ on the $(x, t)$ diagram:

$$
\begin{aligned}
& U_{01}=U_{1}-u_{p} \\
& U_{02}=U_{1} \\
& U_{04}=U_{2} \\
& U_{23}=u_{1,} \cos (\theta-\alpha)
\end{aligned}
$$

where $u_{1 f}$ is the free surface velocity resulting from the reflection of the elastic shock at $\left(x_{2}, t_{2}\right)$ and is given by Fowles [1961] as

$$
u_{1 f}=\frac{u_{1} \sin 2 \alpha}{\sin \theta \cos \alpha+\cos \theta \sin (\alpha-\theta)}
$$

where

$$
\theta=\cos ^{-1}\left\{1-[(2 \nu-1) /(\nu-1)] \sin ^{2} \alpha\right\}
$$

$\nu$ being Poisson's ratio. The angle between the free surface velocity vector and the normal to the impact plane is $\theta-\alpha$. For Lucalox targets, $\nu \simeq 0.236$ [Schreiber and Anderson, 1966], $\theta-\alpha=3.77^{\circ}$, and $U_{23}=2.019 u_{1}$. For $U_{43}$ we use

$$
U_{13}=U_{1}+U_{2 \mathrm{~s}}
$$

which implies that when the material is shocked to the HEL, relieved, and reshocked, the reshocked Hugoniot is the same as the initial Hugoniot. It was found that the computed release wave speeds were relatively insensitive to this assumption (see discussion below).

$$
U_{24}=U_{1}^{\prime} \cos \delta
$$

where $U_{1}^{\prime}$ is the speed of the reflected release wave from point 2 , given by

$$
\begin{aligned}
& U_{1}^{\prime}=U_{1}\left\{\left[1-\frac{u_{1}}{U_{1}} \sin ^{2} \alpha-\left[\left(1-\frac{u_{1}}{U_{1}} \sin ^{2} \alpha\right)^{2}\right.\right.\right. \\
& \left.\left.-\left(1-\frac{u_{1}}{U_{1}}\left(2-\frac{u_{1}}{U_{1}}\right) \sin ^{2} \alpha\right)\left(1-\frac{u_{1}{ }^{2}}{U_{1}^{2}} \cos ^{2} \alpha\right)\right]^{1 / 2}\right] \\
& \left..\left[1-\frac{u_{1}}{U_{1}}\left(2-\frac{u_{1}}{U_{1}}\right) \sin ^{2} \alpha\right]^{-1}\right\}
\end{aligned}
$$

which follows from the assumptions (1) that $\sin \epsilon=\left(U_{1}^{\prime} / U_{1}\right)$ $\sin \alpha$, where $\epsilon$ is the angle of reflection of the elastic shock at the free surface, and (2) that $U_{1}^{\prime}=U_{1}-u_{1} \cos \delta$, where $\delta$ is the angle between the normal to the reflected shock front and the perpendicular to the impact surface (i.e., $\delta=\alpha+\epsilon$ ):

$$
\begin{aligned}
& U_{15}=c+u_{2} \\
& U_{45}=c \cos \delta-u_{2} \\
& U_{58}=c+u_{2}-u_{1}+U_{23}
\end{aligned}
$$

In (14) it is assumed that the speed of the release wave from point 1 is unaltered by interaction with the release wave from point 2. Variations in this assumption have little effect on the computed values of $c$.

$$
U_{57}=\dot{U}_{58}^{\prime}
$$

and

$$
\begin{aligned}
U_{3 \mathrm{a}}= & u_{2}\left(4 \cos ^{2} \alpha+\sin ^{2} \alpha\right)^{1 / 2} \\
& \cdot \cos \left[\tan ^{-1}(2 \cot \alpha)+\alpha-\pi / 2\right]
\end{aligned}
$$

relationships which follow from the assumption that upon reflection the component of $u_{2}$ normal to the free surface is doubled, while the parallel component does not change. For Lucalox targets, $U_{36}=1.821 u_{2}$.

The values of $u_{1}, U_{1}$, and $U_{2}$ were taken from the literature. The value of $u_{2}$ is obtained by an impedance match solution using the measured value of $u_{p}$; for symmetric impacts, $u_{2}=$ $\frac{1}{2} u_{p}$.

Equations (3)-(16) are used to calculate the coordinates $\left(x_{l}\right.$, $t_{\ell}$ ) of the points 1-8 which pertain to a given experiment. Two values of $c$ are computed for each experiment:

1. $L^{*}$ is used to determine $x_{3}$, and a value of $c$ is found such that points 3 and 8 coincide.

2. $L^{* *}$ is used to determine $x_{\mathrm{s}}$, and a value of $c$ is found such that points 6 and 7 coincide.

The results are listed in Table 1 under the headings ' $c\left(L^{*}\right)$ ' and ' $c\left(L^{* *}\right)$,' respectively. The latter value is probably less ieliable, for it is influenced by the assumptions made about free surface velocity in the expressions for $U_{36}$ and $U_{29}$.

At the driving stresses which we produced, there is no elastic precursor in 2024 aluminum. Thus the calculations of release wave speed in the aluminum targets could have proceeded from relatively simple analytic formulas similar to those given by Fowles [1960]. Equivalently, (3)-(16) may be solved with $u_{1}$ set equal to zero. In addition, the identification of $L^{*}$ and $L^{* *}$ proved to be very difficult on the streak records from aluminum targets. However, regions $I$ and 3 could be clearly identified. Therefore we extrapolated straight-line fits to those regions on the streak photographs to obtain the center of region 2, corresponding to a target thickness $L_{a v}$. A value of $c$ was sought such that $\frac{t}{2}\left(L^{*}+L^{* *}\right)=L_{a v}$. For aluminum shot A322, which employed a Lucalox flyer plate, we used a value of $11.50 \mathrm{~km} / \mathrm{s}$ for the release wave speed in the flyer.

The projectiles which we used were constructed of polyethylene and Lexan. Flyer plates thinner than $1.5 \mathrm{~mm}$ were also backed with plates of aluminum about $2 \mathrm{~mm}$ thick. The projectile velocities were computed from travel times between reference laser beams, as was described by Ahrens et al. [1971]. The maximum pressure resulting from the impact was computed by an impedance match solution by using literature data [McQueen et al., 1970; Ahrens et al., 1968]. Specifically, for alumina we fitted the $\sigma_{x}-u_{2}$ data (where $\sigma_{x}$ is the stress in the shock direction) of Ahrens et al. with a line which can be described by $\sigma_{x} / u_{2} \simeq 400 \mathrm{kbar} / \mathrm{km} / \mathrm{s}$; for additional calcu- 
TABLE 1. Release Wave Velocities in Aluminum and Corundum

\begin{tabular}{|c|c|c|c|c|c|c|c|c|c|c|c|c|}
\hline \multirow[b]{2}{*}{ Shot } & \multirow{2}{*}{$\begin{array}{c}\text { Flyer } \\
\text { Material }\end{array}$} & \multirow{2}{*}{$\begin{array}{c}\text { Flyer } \\
\text { Thickness, } \\
\text { mm }\end{array}$} & \multirow{2}{*}{$\begin{array}{c}\text { Target } \\
\text { Material }\end{array}$} & \multirow{2}{*}{$\begin{array}{c}\text { Impact } \\
\text { Velocity, } \\
\mathrm{km} / \mathrm{s}\end{array}$} & \multirow{2}{*}{$\begin{array}{c}\text { Hugoniot } \\
\text { Stress, } \\
\text { kbar }\end{array}$} & \multirow{2}{*}{$\begin{array}{c}\text { Mean } \\
\text { Stress, } \\
\text { kbar }\end{array}$} & \multicolumn{3}{|c|}{$\begin{array}{c}\text { Break Points, } \dagger \\
\text { mm }\end{array}$} & \multirow{2}{*}{$\begin{array}{c}\text { Apparent } \\
\text { Shock } \\
\text { Velocity, } \\
\mathrm{km} / \mathrm{s}\end{array}$} & \multicolumn{2}{|c|}{$\begin{array}{c}\text { Release Wave Speed, } \\
\mathrm{km} / \mathrm{s}\end{array}$} \\
\hline & & & & & & & $L^{*}$ & $L_{a v}$ & $L^{* *}$ & & $c\left(L^{*}\right)$ & $c\left(L^{* *}\right)$ \\
\hline $\begin{array}{l}\text { A318 } \\
\text { A322 }\end{array}$ & $\begin{array}{l}\mathrm{Al} 2024 \\
\mathrm{Al}_{2} \mathrm{O}_{3}\end{array}$ & $\begin{array}{l}2.02 \\
2.54\end{array}$ & $\begin{array}{l}\text { Al } 2024 \\
\text { Al } 2024\end{array}$ & $\begin{array}{l}1.35 \\
1.94\end{array}$ & $\begin{array}{l}117 \\
256\end{array}$ & $\begin{array}{l}114 \\
248\end{array}$ & & $\begin{array}{r}11.72 \\
10.76 \\
9.65\end{array}$ & & $\begin{array}{l}9.24 \\
8.24\end{array}$ & $\begin{array}{c}7.83 \pm 0.15 \\
7.83 \\
8.14 \text { or } \\
>8.79 \ddagger\end{array}$ & \\
\hline $\begin{array}{l}\text { A323 } \\
\text { A327 }\end{array}$ & $\begin{array}{l}\mathrm{Al}_{2} \mathrm{O}_{3} \\
\mathrm{Al}_{2} \mathrm{O}_{3}\end{array}$ & $\begin{array}{l}2.52 \\
2.59\end{array}$ & $\begin{array}{l}\mathrm{Al}_{2} \mathrm{O}_{3} \\
\mathrm{Al}_{2} \mathrm{O}_{3}\end{array}$ & $\begin{array}{l}1.98 \\
1.99\end{array}$ & $\begin{array}{l}395 \\
396\end{array}$ & $\begin{array}{l}352 \\
353\end{array}$ & $\begin{array}{l}15.00 \\
10.50\end{array}$ & & $\begin{array}{r}14.54 \\
9.00\end{array}$ & $\begin{array}{l}10.76 \\
10.52\end{array}$ & $11.90 \pm 0.05$ & $11.35 \pm 0.05$ \\
\hline A333 & $\mathrm{Al}_{2} \mathrm{O}_{3}$ & 2.03 & $\mathrm{Al}_{2} \mathrm{O}_{3}$ & 1.78 & 355 & 312 & 13.00 & & 10.34 & 11.50 & $11.52 \pm 0.07$ & $11.26 \pm 0.10$ \\
\hline A344 & $\mathrm{Al}_{2} \mathrm{O}_{3}$ & 2.03 & $\mathrm{Al}_{2} \mathrm{O}_{3}$ & 1.91 & 390 & 347 & 11.79 & & 8.79 & 11.00 & $11.95 \pm 0.07$ & $11.82 \pm 0.10$ \\
\hline A351 & $\mathrm{Al}_{2} \mathrm{O}_{3}$ & 1.39 & $\mathrm{Al}_{2} \mathrm{O}_{3}$ & 1.01 & 205 & 162 & 10.48 & & 9.58 & 10.62 & $10.65 \pm 0.02$ & $9.49 \pm 0.02$ \\
\hline A352 & $\mathrm{Al}_{2} \mathrm{O}_{3}$ & 1.27 & $\mathrm{Al}_{2} \mathrm{O}_{3}$ & 1.00 & 204 & 161 & 10.87 & & 9.01 & 8.62 & $10.35 \pm 0.04$ & $9.30 \pm 0.02$ \\
\hline A353 & $\mathrm{Al}_{2} \mathrm{C}$ & 1.54 & $\mathrm{Al}_{2} \mathrm{O}_{3}$ & 1.45 & 285 & 242 & 11.14 & & 8.09 & 8.95 & $10.97 \pm 0.03$ & $10.40 \pm 0.05$ \\
\hline A355 & $\mathrm{Al}_{2}$ & 2.57 & $\mathrm{Al}_{2}$ & 1.97 & 394 & 351 & 12.64 & & 10.22 & 11.81 & $12.71 \pm 0.13$ & $12.58 \pm 0.12$ \\
\hline
\end{tabular}

$\dagger L_{a v}$ is for aluminum targets, and $L^{*}$ is for $\mathrm{Al}_{2} \mathrm{O}_{3}$ targets.

$\$$ The interpretation of the streak record for this shot was ambivalent (see text).

lations we assumed $U_{1}=10.94 \mathrm{~km} / \mathrm{s}, u_{1}=0.228 \mathrm{~km} / \mathrm{s}$, and $\dot{U}_{2}$ $=1.8 u_{2}+7.8$ (in kilometers per second). For aluminum we employed $U=5.328+1.338 u$.

The pressure associated with the release wave was also estimated by an impedance match technique. For Lucalox flyer plates the rarefaction wave from point 1 in Figure 2 amounts to $\sim 65 \mathrm{kbar}$ for plastic backing and to $\sim 35 \mathrm{kbar}$ for aluminum backing.

\section{RESULTS OF EXPERIMENTS}

The results of the experiments with aluminum and alumina targets are given in Table 1. The uncertainties given for values of $c$ arise from errors in the measurement of $L^{*}$ and $L^{* *}$. The results for alumina are also subject to possible systematic errors due to uncertainty in the value of $U_{2}$. Since the shock velocities calculated from $U$ (apparent shock speed) for the aluminum and alumina targets scatter about the literature values by $10-20 \%$, we were not able to improve upon the $\pm \sim 1 \%$ precision in $U_{2}$ of Ahrens et al. [1968]. This degree of precision results in a maximum uncertainty in release speeds of $\pm 2 \%$. As was discussed above, the value of $c$ computed from $L^{* *}$ (region 1/region 2) is subject to additional systematic error due to the approximations used for computing the free surface velocities, $U_{23}$ and $U_{36}$. If an estimated $10 \%$ possible error in the computed value of the normal component of the free surface velocity is assumed, the uncertainty in $c\left(L^{* *}\right)$ is $2-3 \%$. No $c$ data are given for shot A327 because the target thickness in that experiment was too thin to yield good measurements of $L^{* *}$.

Additional possible systematic error arises from the assumptions used in evaluating $U_{48}$ and $U_{68}$. The uncertainties associated with the value of $U_{43}$ were investigated by trying the alternate assumption that $U_{43}=U_{2}+U_{29}$. The data of Ahrens et al. were reanalyzed with this relationship, and the $U_{2}-u_{2}$ relationship for Lucalox became $U_{2}=8.1+1.617 u_{2}$. However, the effect on $c\left(L^{*}\right)$ was small: the values were shifted upward $\sim 0.02 \mathrm{~km} / \mathrm{s}$ at $\sim 200-\mathrm{kb}$ ar driving stress and $\sim 0.05 \mathrm{~km} / \mathrm{s}$ at $\sim 400$-kbar driving stress. The consequences of a variation in $U_{58}$ were explored by substituting $v_{p}$ (at zero pressure) for $c$ in (14). The effect on $c\left(L^{*}\right)$ was variable, the absolute value of the change in $c$ being less than $0.1 \mathrm{~km} / \mathrm{s}$ in all cases except shot A355, for which $c\left(L^{*}\right)$ was increased by 0.115 $\mathrm{km} / \mathrm{s}$.

The error in Hugoniot stress $\sigma_{x}$ for Lucalox is everywhere less than $\pm 5 \mathrm{kbar}$, the major uncertainty arising from lack of definition of the $\sigma_{x}-u_{2}$ Hugoniot as determined by Ahrens et al. Mean stress values for Lucalox are computed from

$$
P=\sigma_{x}-\frac{4}{3} \frac{\mu \sigma_{\mathrm{HEL}}}{K+(4 / 3) \mu}
$$

where $\mu$ is the shear modulus and $\sigma_{\mathrm{HEL}}$ is the stress at the HEL. The elastic constants are evaluated at the HEL by means of the pressure derivatives determined by Schreiber and Anderson [1966]. Values of Hugoniot stress for aluminum are accurate to less than a few kilobars. Mean stress for the aluminum shots was computed from previous determinations of the dynamic von Mises limit for 2024-T4 aluminum [Fowles, 1961; Kusubov and van Thiel, $1969 a, b]$; the uncertainty in $P-\sigma_{x}$ is about 0.6 kbar for $\sigma_{x}=117 \mathrm{kbar}$ and $2 \mathrm{kbar}$ for $\sigma_{x}=255 \mathrm{kbar}$.

The inability to resolve $L^{*}$ and $L^{* *}$ contributed a small error to the values for the aluminum shots, less than 0.5 and $0.8 \%$ for shots $A 318$ and $A 322$, respectively. Owing to the thickness of the A322 target, arrivals from release waves with speeds greater than $8.76 \mathrm{~km} / \mathrm{s}$ merged with rarefaction waves from the edge of the flyer plate and thus could not be resolved. Moreover, in shot A322 there is additional uncertainty in $c$ due to uncertainty in the release speed in the flyer. The values in Table 1 were derived with $c$ (flyer) $=11.5 \mathrm{~km} / \mathrm{s}$. It is apparent from Figure 5 that this value is uncertain by possibly as much at $\pm 0.5 \mathrm{~km} / \mathrm{s}$, which contributes a $\pm 10 \%$ uncertainty to the calculated values of $c$ for shot $\mathrm{A} 322$.

\section{Discussion OF RESULTS}

In Figure 4 the results of the two shots with aluminum

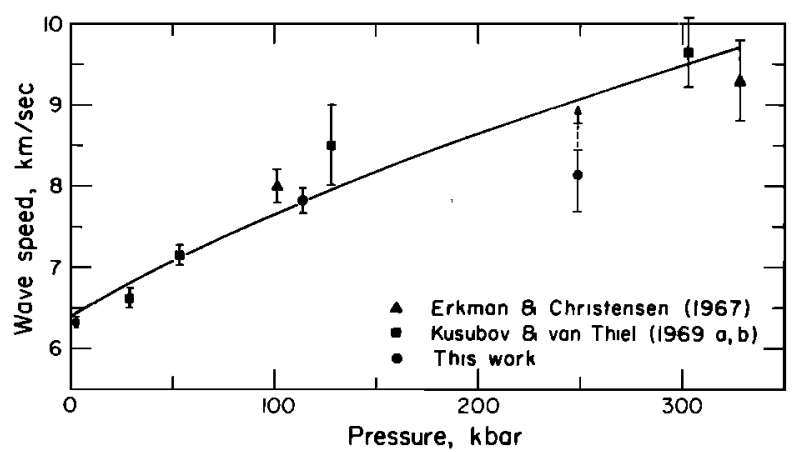

Fig. 4. Release wave speed in 2024 aluminum. Fourth-order finite strain extrapolation of $V_{p}$ for $\partial^{2} M / \partial P^{2}=0.00275 \mathrm{kbar}^{-1}\left(M=\rho v_{p}{ }^{2}\right)$ is shown. 
targets are compared with data taken from the literature. The agreement is quite good for shot A318 and fair for the relatively uncertain data from shot A322.

Fourth-order hydrostatic finite strain extrapolations [Davies and Ahrens, 1973; Davies, 1974] were carried out for the elastic wave speed in aluminum, as is also shown in Figure 4. According to this formulation the longitudinal modulus $M$ (equal to $\left.\rho V_{p}^{2}\right)$ is given by

$$
M=\rho_{0}(1-2 \epsilon)^{7 / 2} \cdot\left(r_{11}{ }^{0}+r_{11}^{1} \epsilon+\frac{1}{2} r_{11}{ }^{2} \epsilon^{2}\right)-P \Delta_{11}
$$

and the pressure $\boldsymbol{P}$ is found from

$$
P=\$ \rho_{0}(1-2 \epsilon)^{6 / 2}\left(C_{0}+C_{1} \epsilon+C_{2} \epsilon^{2}+C_{3} \epsilon^{3}\right)
$$

where the Eulerian strain parameter $\epsilon$ is related to actual density $\rho$ and initial density $\rho_{0}$ by

$$
\epsilon=\frac{1}{2}\left[1-\left(\rho / \rho_{0}\right)^{2 / 3}\right]
$$

and for cubic or isotropic symmetry $\Delta_{11}=-3$. The finite strain coefficients are evaluated from

$$
C_{0}=-3 P_{0} / \rho_{0}
$$

where $P_{0}$ is the initial pressure and

$$
\begin{aligned}
C_{1} & =3\left(r_{11}{ }^{0}+2 r_{12}{ }^{0}\right) \\
C_{2} & =\frac{3}{2}\left(r_{11}{ }^{1}+2 r_{12}{ }^{1}\right) \\
C_{3} & =\frac{1}{2}\left(r_{11}{ }^{2}+2 r_{12}{ }^{2}\right) \\
r_{1 i}{ }^{0} & =\left(C_{1 i}+P_{0} \Delta_{i i}\right) / \rho_{0} \\
r_{i i}{ }^{1} & =-3 K_{0}\left(C_{1 i}{ }^{\prime}+\Delta_{i i}\right) / \rho_{0}+7 r_{i i}{ }^{0} \\
r_{1 i}{ }^{2} & =9 K_{0}{ }^{2} C_{i i}{ }^{\prime \prime} / \rho_{0}-3 K_{0}{ }^{\prime}\left(r_{1 i}{ }^{1}-7 r_{i i}{ }^{0}\right) \\
& \quad+16 r_{i j}{ }^{1}-49 r_{i j}{ }^{0}
\end{aligned}
$$

For an isotropic solid, $\Delta_{12}=-1, \Delta_{44}=-1, C_{11}=M=K+$ qu, and $C_{12}=K-\frac{2}{\xi} \mu$. Parameter $\mu$ is the shear modulus, and the primes denote differentation with respect to pressure. We have not explored the implications of the present experimental results in terms of alternate (Lagrangian) finite strain formulations [Thomsen, 1972].

Values for the first pressure derivatives of the elastic moduli were computed from data compiled by Simmons and Wang [1971]; $\partial K / \partial P=3.0$, and $\partial \mu / \partial P=2.82$. Weighted least squares fitting [cf. Deming. 1943], using the data above $5 \mathrm{kbar}$, gives $\partial^{2} M / \partial P^{2}=0.0028 \pm 0.0054 \mathrm{kbar}^{-1}$ when $\partial^{2} K / \partial P^{2}=$ $\partial^{2} M / \partial P^{2}$ and $\partial^{2} M / \partial P^{2}=0.002 \pm 0.004 \mathrm{kbar}^{-1}$ when $\partial^{2} K / \partial P^{2}$ $=0$. Thus $M\left(\partial^{2} M / \partial P^{2}\right)$ for aluminum has the value $\sim 2.7$ \pm 5.7. Except possibly for the region $\lesssim 30 \mathrm{kbar}$ the theory accounts quite well for the observed release wave speeds.

In Figure 5 the data for rarefaction wave speed in Lucalox are compared with extrapolations of the longitudinal and bulk elastic velocities, also based on the fourth-order finite strain theory developed by Davies ((18)-(27)). In applying this theory to Lucalox, which is shocked to a region where shear strains are still significant, we make the assumption that the elastic wave speeds are solely functions of mean stress. We have plotted the values of $c$ determined from $L^{*}$, since we feel that those values are more reliable. The finite strain formulas were evaluated by using ultrasonic data of Anderson et al. [1968]: values at $20^{\circ} \mathrm{C}$ and zero pressure were $K_{0}=2521$ kbar, $\mu_{0}=1613 \mathrm{kbar}, \partial K / \partial T=-0.14 \mathrm{bar} /{ }^{\circ} \mathrm{C}, \partial \mu_{0} / \partial T=-0.18$ $\mathrm{kbar} /{ }^{\circ} \mathrm{C}, \partial K / \partial P=3.98, \partial \mu / \partial P=1.76$, and $\rho_{0}=3.98 \mathrm{~g} / \mathrm{cm}^{3}$.
A correction was made for the difference between the Hugoniot temperature (taken at $\mathbf{4 0 0}$ kbar from the calculations of Ahrens et al. [1968]) and the adiabatic temperature (calculated on the assumption that the Gruneisen constant divided by the specific volume is constant) by using zero-pressure moduli evaluated at $75^{\circ} \mathrm{C}$. Between 360 and 400 kbar the rarefaction wave speeds are in general agreement with longitudinal and sound speeds extrapolated with values of $\partial^{2} M / \partial P^{2}$ of about $-0.01 \mathrm{kbar}^{-1}$. Bounds on the value of $\partial^{2} M / \partial P^{2}$ were obtained by calculating the least squares fit to all the data under the assumptions $\partial^{2} K / \partial P^{2}=\partial^{2} M / \partial P^{2}$ and $\partial^{2} K / \partial P^{2}=0$. The results are $\partial^{2} M / \partial P^{2}=-0.0127$ and $-0.0143 \mathrm{kbar}^{-1}$, respectively.

These values are strongly influenced by the three data points which lie below 300-kbar driving stress, which, as is discussed below, may not represent longitudinal elastic wave speeds. A fit to the four higher pressure points, using the same bounding procedure, gives $-0.0102<\partial^{2} M / \partial P^{2}<-0.0097 \mathrm{kbar}^{-1}$. The computed standard error for the bounding values is $\sim 0.005$ $\mathrm{kbar}^{-1}$, but with such a small data base ( 3 degrees of freedom) this estimate is only qualitative.

The hydrostatic data of Hart and Drickamer [1968], which extend to only $304 \mathrm{kbar}$, imply that $\partial^{2} K / \partial P^{2} \gtrsim-0.002$ $\mathrm{kbar}^{-1}$.

We note that these results are similar to those of Graham [1972], who studied the shock compression of single-crystal $\mathrm{Al}_{2} \mathrm{O}_{3}$ below the HEL in the (100) and (001) directions. He found no sensible difference in moduli along these directions. His value of $C_{x x}$ at $\sigma_{x} \simeq 200 \mathrm{kbar}$ ( $\sim 118$-kbar mean stress), when it is compared with the zero-pressure values of $C_{11}$ and $C_{33}$ extrapolated with the mean values of $\partial C_{x x} / \partial P$ determined ultrasonically by Hankey and Schuele [1970], implies that $C_{x x}\left(\partial^{2} C_{x x} / \partial P\right)$ is $-26 \pm \sim 75$ for $x x=11$ (transverse to the principal axis) and $43 \pm \sim 75$ for $x x=33$ (parallel to the principal axis). By comparison, we find that the value of $M\left(\partial^{2} M / \partial P^{2}\right) \approx-46 \pm \sim 24$. Since $M$ is a function of all six independent second-order single-crystal elastic constants, its derivatives cannot be directly compared with those of $C_{x x}$; however, longitudinal moduli in single-crystal $\mathrm{Al}_{2} \mathrm{O}_{3}$ vary little with direction (e.g., $C_{11} / C_{39}=0.997$ ), so that rough agreement between these two measurements is to be expected. Since the transverse moduli play a more important role in determining bulk properties than the parallel moduli, the closer agreement with $C_{11}\left(\partial^{2} C_{11} / \partial P^{2}\right)$ is also to be expected.

The relatively low values of release wave speed measured at 200 and 285 kbar deserve additional comment. There are four possible explanations for these data:

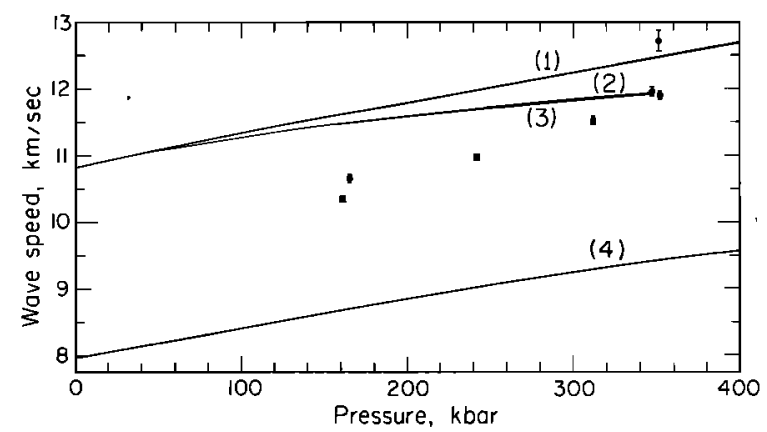

Fig. 5. Release wave speeds in alumina. Fourth-order finite strain extrapolations of $V_{p}$ and bulk sound speed are shown: 1-3, longitudinal wave speed, with $\partial^{2} K / \partial P^{2}=\partial^{2} M / \partial P^{2}=0, \partial^{2} K / \partial P^{2}=\partial^{2} M / \partial P^{2}$ $=-0.0097 \mathrm{kbar}^{-1}$, and $\partial^{2} M / \partial P^{2}=\mathbf{f}\left(\partial^{2} \mu / \partial P^{2}\right)=-0.0102 \mathrm{kbar}^{-1}$, respectively; and 4 , bulk sound speed, with $\partial^{2} K / \partial P^{2}=0$. 
1. The shear modulus may decrease upon shear failure. This effect has been observed in quartzose rock, in which melting along failure planes has been postulated [Grady et al., 1975]. Relatively low release wave speeds have also been observed in polycrystalline iron [Barker and Hollenbach, 1974]. The data for $\alpha$ iron are compared with extrapolations of elastic wave speeds (based on data from Guinan and Beshers [1968]: $K_{0}=1664 \mathrm{kbar}, \mu_{0}=814 \mathrm{kbar}, \partial K / \partial P=5.29$, and $\partial \mu / \partial P=$ 1.85 ) in Figure 6. Agreement is obtained only for extraordinarily negative values of $\partial^{2} M / \partial P^{2}$, e.g., $-0.043 \mathrm{kbar}^{-1}$. These observations may reflect a decrease in $\mu$ as a phase transition is approached, as has been proposed by Anderson [1972]. Graham and Brooks [1971] also observed a very large decrease in the shear modulus of single-crystal $\mathrm{Al}_{2} \mathrm{O}_{3}$ above the HEL. However, the single crystals fail at $\sigma_{x}=120-210 \mathrm{kbar}$, a finding which implies shear stresses that (according to von Mises yield theory) are never reached in polycrystalline Lucalox. (Evidently, the failure mechanisms differ in these two materials.) Moreover, Ahrens et al. found a significant offset between the Hugoniot and the hydrostat (the mean stress versus volume curve) of alumina, from which it was inferred that the shear modulus is nearly constant.

2. The elastic-plastic release model may not apply to alumina shocked in this pressure range. A similar conclusion was reached for $\epsilon$ iron and possibly for $\alpha$ iron relieved from stress states of 100-400 kbar [Barker and Hollenbach, 1974]. In aluminum the elastic and plastic release waves also fail to separate, as would be predicted by elastic-plastic theory [Erkman and Christensen, 1967].

3. The shots in which low release wave speeds were observed were also those in which the Lucalox flyer plates were backed with aluminum support discs; it may be that because the release waves were of lower amplitude, the first arrivals produced no significant effect on the streak records.

4. The release wave speeds might truly represent the longitudinal sound speeds in the material were it under an equivalent hydrostatic pressure. This would imply either that $\partial V_{p} / \partial P$ approaches zero or becomes negative below $200 \mathrm{kbar}$ or that the zero-pressure value of $\partial V_{p} / \partial P$ is seriously in error. The stability of shock waves at stresses below $200 \mathrm{kbar}$ and the consistency of measured values of $\partial V_{p} / \partial P$ for alumina and other oxides appear to contradict both of these inferences.

We favor points 1 or 2 above as the probable explanation of our data. It seems conceivable that whatever mechanism causes the catastrophic loss of shear strength in single-crystal aluminum oxide may also be activated during the release process in polycrystalline material. The approach of the release wave speed to reasonable values of $V_{p}$ at higher pressure may indicate increasing shear strength with compression. Thus the value of $\partial^{2} M / \partial P^{2}$ derived from our experiments on Lucalox should be considered a lower bound.

\section{CONCLUSIONS}

The agreement of rarefaction wave speed in shock-compressed aluminum and in polycrystalline alumina shocked to stresses above $\sim 350 \mathrm{kbar}$ with extrapolations of $V_{p}$ using fourth-order finite strain theory points to initially elastic unloading behavior in these materials at these stresses. Values for $M\left(\partial^{2} M / \partial P^{2}\right)$ of $\sim 2.7 \pm 5.7$ for aluminum and $\sim-50$ for alumina can thus be obtained from the shock wave data. The value of $M\left(\partial^{2} M / \partial P^{2}\right)$ for alumina is in agreement with values of $C_{x x}\left(\partial^{2} C_{x x} / \partial P^{2}\right)$ measured previously for single-crystal aluminum oxide.

In alumina shocked to stresses of $200-300 \mathrm{kbar}$, as in alumi-

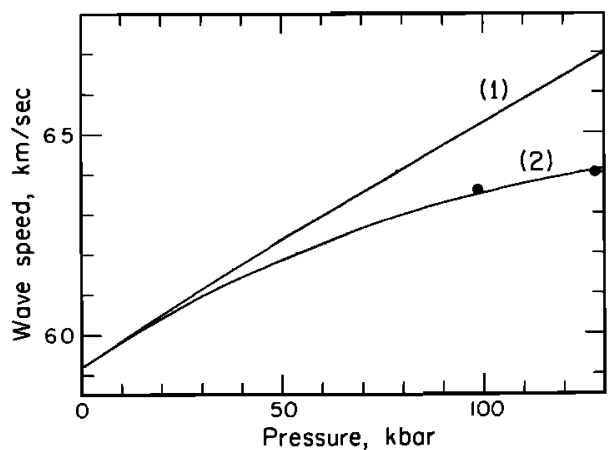

Fig. 6. Release wave speeds measured in $\alpha$ iron by Barker and Hollenbach [1974], compared with fourth-order finite strain extrapolations of $V_{p}: 1, \partial^{2} M / \partial P^{2}=\partial^{2} K / \partial P^{2}=0$; and $2, \partial^{2} M / \partial P^{2}=$ $\partial^{2} K / \partial P^{e}=-0.0425 \mathrm{kbar}^{-1}$.

num shocked below $30 \mathrm{kbar}$ and iron shocked to above 100 kbar, the release wave speeds do not agree with any reasonable extrapolation of either elastic wave speed. Deviation from elastic-plastic release behavior is considered the most likely explanation of this discrepancy, except in iron shocked to $\sim 100 \mathrm{kbar}$, to which a decrease in the shear modulus in the neighborhood of a phase transition may also be a contributing factor.

Acknowledgments. This research was supported under NSF grant GA $39042 X$. The authors appreciate the conscientious operation of the gun facility by Harold Richeson and David Johnson. Comments of $M$. van Thiel have also been very valuable to us. Contribution 2632 of the Division of Geological and Planetary Sciences, California Institute of Technology, Pasadena, California.

\section{REFERENCES}

Ahrens, T. J., The mineralogic distribution of iron in the upper mantle, Phys. Earth Planet. Interiors, 5, 267-281, 1972.

Ahrens, T. J., and L. Thomsen, Application of the fourth order anharmonic theory to the prediction of equation of state at high compression and temperatures, Phys. Earth Planet. Interiors, 5 , 282-294, 1972.

Ahrens, T. J., W. H. Gust, and E. B. Royce, Material strength effect in the shock compression of alumina, J. Appl. Phys., 39, 4610-4616, 1968.

Ahrens, T. J., J. H. Lower, and P. Lagus, Equation of state of forsterite, J. Geophys. Res., 76, 518-527, 1971.

Anderson, D. L., A seismic equation of state, Geophys. J. Roy. Astron. Soc., 13, 9-30, 1967.

Anderson, O. L., Shock wave determination of the shear velocity at very high pressures, Phys. Earth Planet. Interiors, 6, 136-140, 1972.

Anderson, O. L., E. Schreiber, R. C. Liebermann, and N. Soga, Some elastic constant data on minerals relevant to geophysics, Rev. Geophys. Space Phys., 6, 491-524, 1968.

Barker, L. M., and R. E. Hollenbach, Shock wave study of the $\alpha=\epsilon$ phase transition in iron, J. Appl. Phys., 45, 4872-4887, 1974.

Birch, F., Elasticity and constitution of the earth's interior, $J$. Geophys. Res., 57, 227-286, 1952.

Burdick, L., and D. L. Anderson, Interpretation of velocity profiles of the mantle, J. Geophys. Res., 80, 1070-1074, 1975.

Davies, G. F., Effective elastic moduli under hydrostatic stress, 1 , Quasi-harmonic theory, J. Phys. Chem. Solids, 35, 1513-1520, 1974.

Davies, G. F., and T. J. Ahrens, Measurements of elastic velocities of $\mathrm{MgO}$ under shock compression to $500 \mathrm{kbar}, J$. Geophys. Res., 78, 7596-7601, 1973.

Deming, W. E., Statistical Adjustment of Data, John Wiley, New York, 1943. (Also Dover, New York, 1966.)

Erkman, J. O., and A. B. Christensen, Attenuation of shock waves in aluminum, J. Appl. Phys., 38, 5395-5403, 1967.

Fowles, G. R., Attenuation of the shock wave produced in a solid by a flying plate, J. Appl. Phys., 3l, 655-661, 1960.

Fowles, G. R., Shock wave compression of hardened and annealed 2024 aluminum, J. Appl. Phys., 32, 1475-1487, 1961.

Grady, D., W. Murri, and P. De Carli, Hugoniot sound velocities and 
'.

phase transformations in two silicates, J. Geophys. Res., 80, 4857, 1975.

Graham, R. A., Determination of third- and fourth-order longitudinal elastic constants by shock compression techniques: Application to sapphire and fused quartz, J. Acoust. Soc. Amer., 5I, 1576-1581, 1972.

Graham, R. A., and W. P. Brooks, Shock wave compression of sapphire from 15 to $\mathbf{4 2 0} \mathrm{kbar}$ : The effects of large anisotropic compressions, J. Phys. Chem. Solids, 32, 2311-2330, 1971.

Guinan, M., and D. N. Beshers, Pressure derivatives of the elastic constants of $\alpha$-iron to 10 kbars, J. Phys. Chem. Solids, 29, 541-549, 1968.

Hankey, R. E., and D. E. Schuele, Third-order elastic constants of $\mathrm{Al}_{2} \mathrm{O}_{3}$, J. Acoust. Soc. Amer., 48, 190-202, 1970.

Hart, H. V., and H. G. Drickamer, Effect of high pressure on lattice parameters of $\mathrm{Al}_{2} \mathrm{O}_{8}$, J. Chem. Phys., 43, 2265-2266, 1968.

Kusubov, A. S., and M. van Thiel, Measurement of elastic and plastic unloading wave profiles in 2024-T4 aluminum alloy, J. Appl. Phys., 40, 3776-3780, 1969a.

Kusubov, A. S., and M. van Thiel, Dynamic yield strength of 2024-T4 aluminum at $313 \mathrm{kbar}, J$. Appl. Phys., 40, 893-894, $1969 \mathrm{~b}$.

McQueen, R. G., S. P. Marsh, J. W. Taylor, J. N. Fritz, and W. J. Carter, The equation of state of solids from shock wave studies, in High Velocity Impact Phenomena, edited by R. Kinslow, pp. 294-419, Academic, New York, 1970.
Sammis, C., Lattice dynamics of $\mathrm{MgO}_{1} \mathrm{Al}_{2} \mathrm{MgO}_{4}$, and $\mathrm{Mg}_{2} \mathrm{SiO}_{4}$ spinel, Geophys. J. Roy. Astron. Soc., 29, 15-42, 1972.

Schreiber, E., and O. L. Anderson, Pressure derivatives of the sound velocities of polycrystalline alumina. J. Amer. Ceram. Soc., 49, 184-189, 1966.

Simmons, G., and H. Wang, Single Crystal Elastic Constants and Calculated Aggregate Properties: A Handbook, 2nd ed., MIT Press, Cambridge, Mass., 1971.

Soga, N., and O. L. Anderson, High temperature elasticity and expansivity of forsterite and steatite, J. Amer. Ceram. Soc., 50, 239-292, 1967.

Spetzler, H., Equation of state of polycrystalline and single-crystal MgO to 8 kbar and $800^{\circ} \mathrm{K}$, J. Geophys. Res., 75, 2073-2087, 1970.

Thomsen, L., The fourth-order anharmonic theory: Elasticity and stability, J. Phys. Chem. Solids, 33, 363-378, 1972.

Zel'dovich, Y. B., and Y. P. Raizer, Physics of Shock Waves and High Temperature Hydrodynamic Phenomena. Academic, New York, 1966.

(Received May 27, 1975;

revised August 26, 1975;

accepted September 5, 1975.) 